\title{
Quelques représentations d'embarcations monoxyles en Asie du Sud-Est
}

Several descriptions of the monoxyla boats of Southeast Asia

Algunas representaciones de embarcaciones monoxilas en Asia del Sudeste

\section{Michel Jacq-Hergoualc'h}

\section{(2) OpenEdition}

Journals

Édition électronique

URL : https://journals.openedition.org/tc/302

DOI : $10.4000 /$ tc. 302

ISSN : 1952-420X

Éditeur

Éditions de l'EHESS

Édition imprimée

Date de publication : 1 janvier 2001

Pagination : 401-416

ISSN : 0248-6016

Référence électronique

Michel Jacq-Hergoualc'h, «Quelques représentations d'embarcations monoxyles en Asie du Sud-Est », Techniques \& Culture [En ligne], 35-36 | 2001, mis en ligne le 10 septembre 2012, consulté le 29

septembre 2022. URL : http://journals.openedition.org/tc/302 ; DOI : https://doi.org/10.4000/tc.302

Ce document a été généré automatiquement le 29 septembre 2022.

Tous droits réservés 


\title{
Quelques représentations d'embarcations monoxyles en Asie du Sud-Est
}

\author{
Several descriptions of the monoxyla boats of Southeast Asia \\ Algunas representaciones de embarcaciones monoxilas en Asia del Sudeste
}

Michel Jacq-Hergoualc'h

1 Les représentations de barques monoxyles qui apparaissent sur les tambours de bronze de la civilisation Dông So'n (Viêt Nam) sont les plus anciennes, mais elles sont très stylisées. Cette particularité a donné libre cours à de nombreuses interprétations au fil des études sur lesdits tambours, ceux-ci étant eux-mêmes l'objet des appréciations les plus délirantes. Nous avons donc choisi d'évoquer d'abord certaines des barques des bas-reliefs khmers -en l'occurrence les barques de guerre-, en raison du relatif réalisme qui présida à leur représentation (Jacq-Hergoualc'h 1979 : 165-182, 15 doc).

2 Isolées ou prenant part à un combat naval, elles s'y retrouvent par deux fois. Au Bàyon, d'une part, sur le mur de fond de la galerie extérieure, face sud, aile est, scène dont il faut rapprocher des figurations localisées dans la même aile mais un peu plus à l'ouest, et celles qui sont figurées sur certains panneaux de l'angle sud-est de la même galerie ; d'autre part, à Banteay Chmar où l'on découvre la représentation d'une nouvelle bataille navale sur tout un panneau de la galerie extérieure est, aile sud. Ces deux grandes batailles seront l'objet principal de nos remarques et nous négligerons toutes les embarcations qui peuvent figurer sur d'autres bas-reliefs des mêmes temples ou d'autres sanctuaires mais qui indiquent un usage civil.

3 Sur les deux ensembles, la flotte khmère est opposée à la flotte came. Cette dernière a nettement le dessous dans des combats qui font rage. Au Bàyon, ses embarcations sont envahies par les guerriers khmers; l'une d'elles est même en train de couler et les Cams sont précipités à l'eau de toutes parts; les pertes qu'ils infligent aux Khmers sont minimes. À Banteay Chmar, le scénario est identique. Les Cams sont en plein désarroi ; leurs chefs font le geste de s'enfuir et les rameurs qui, habituellement, rament face à 
l'arrière ont tous inversé leur position, comme s'ils voulaient dégager leurs embarcations et fuir au plus vite ; leurs pertes sont considérables.

Ces deux batailles, décrites par de nombreux auteurs, ont été rapprochées l'une de l'autre, et G. Cœdès en 1929 puis en 1932 proposa d'y voir la figuration de la victoire navale que Jayavarman VII remporta sur les Cams envahisseurs peu après 1177, victoire qui mit fin à leur occupation du Cambodge et préluda au relèvement du pays sous le gouvernement avisé du nouveau roi. Cette bataille n'a pu avoir lieu qu'aux alentours du centre vital du pays, autrement dit d'Angkor ; elle est donc bien fluviale et s'est peutêtre déroulée sur le Tonlé Sap.

Dans les deux batailles, du côté des Khmers, 29 bateaux sont représentés de façon plus ou moins complète. Parfois, seul le corps de la barque apparaît. Cependant, sur ces 29 bateaux, 26 ont au moins leur proue ou leur poupe visible et 15 sont complets. Ces embarcations ont évidemment des caractères communs : elles sont basses sur l'eau et de forme allongée, « large au centre et effilées aux deux bouts » comme l'indique Zhou Daguan (Tcheou Ta-kouan) (Pelliot 1902: 172); «Elles n'ont pas de voiles et peuvent porter plusieurs personnes; on ne les dirige qu'à la rame ", précise encore le même auteur chinois. Il faut ajouter que, parfois, les rameurs sont remplacés par des pagayeurs, les uns comme les autres étant répartis en deux rangées symétriques; elles sont dirigées par un nautonier, placé à l'arrière et doté d'une grande rame en guise de gouvernail ; enfin, elles sont toutes chargées de guerriers prêts au combat.

6 Les différences apparaissent surtout au niveau de leur décoration sculptée de proue et de poupe et, accessoirement, dans la manière dont elles sont propulsées et protégées. Nous avons différencié six types d'embarcations khmères à partir de ces critères, du premier surtout. Dans la description de ces types, nous ne reviendrons pas sur les caractères généraux déjà évoqués, Il suffira d'insister sur ce qui fait l'originalité de chacun.

7 Le type I est le plus simple (fig. 1); il ne comporte aucune décoration de proue ou de poupe ; celles-ci sont lisses. Il n'est représenté que par une embarcation complète et une proue isolée.

8 Sur le second type (fig. 2), apparait une décoration de proue consistant en une tête de makara, gueule ouverte, dotée de défenses saillantes qui jouent peut-être le rôle d'éperons; mais la partie supérieure de la proue se relève, lisse comme la poupe. C'est aussi un type peu représenté : une embarcation complète et deux proues isolées.

9 Le type III (fig. 3) n'apparaît dans aucune des deux batailles mais est figuré sur les panneaux de l'angle sud-est de la galerie extérieure du Bàyon. La proue a perdu son makara mais se redresse en une fière tête de garuda surmontée d'oriflammes. La poupe est relevée et sculptée en ce que nous pouvons appeler une queue de naga ou peut-être aussi de garuda. Ce type est représenté par deux embarcations complètes et par six proues - toutes identiques.

10 Le type IV (fig. 4) est l'un des plus riches et des plus représentés. En proue se retrouve le makara sculpté figuré sur le type II mais il crache en plus une tête de hamsa. En poupe, on découvre un motif comparable à celui du type III. Six embarcations entières représentent ce type ; il faut y ajouter deux proues du même genre.

11 Il existe, outre ces quatre types, deux embarcations exceptionnelles, l'une au Bàyon, l'autre à Banteay Chmar, qui semblent appartenir au commandant en chef des forces navales khmères, Jayavarman VII. Nous ne possédons toujours pas de bonnes 
photographies de celle représentée à Banteay Chmar, et celle du Bàyon est incomplète, sa partie supérieure ayant disparu. défenses très développées qui ont une véritable allure d'éperons. Par ailleurs, cet animal crache ce qui doit être un naga polycéphale au lieu d'un garuda. En poupe, l'embarcation supporte une cabine couverte assez richement décorée, notamment d'une frise d'animaux atlantes. Devant cette cabine, se tient le nautonier. En son centre, enfin, elle parait comporter une plate-forme où devait prendre place le chef, comme nous allons le voir à Banteay Chmar.

embarcation (type VI, fig. 6) comporte en proue le même makara que précédemment, aux défenses en éperons, crachant le même naga, mais les têtes multiples de celui-ci sont écartées par une figure féminine mitrée. G. Groslier (1921: 110, fig. $72 \mathrm{G}$ ) en a donné un croquis approximatif. La poupe se recourbe en une queue de naga. Au centre existe une plate-forme à balustrade dont les extrémités s'achèvent en tête de naga et qui supporte le chef, cuirassé et armé de son arc.

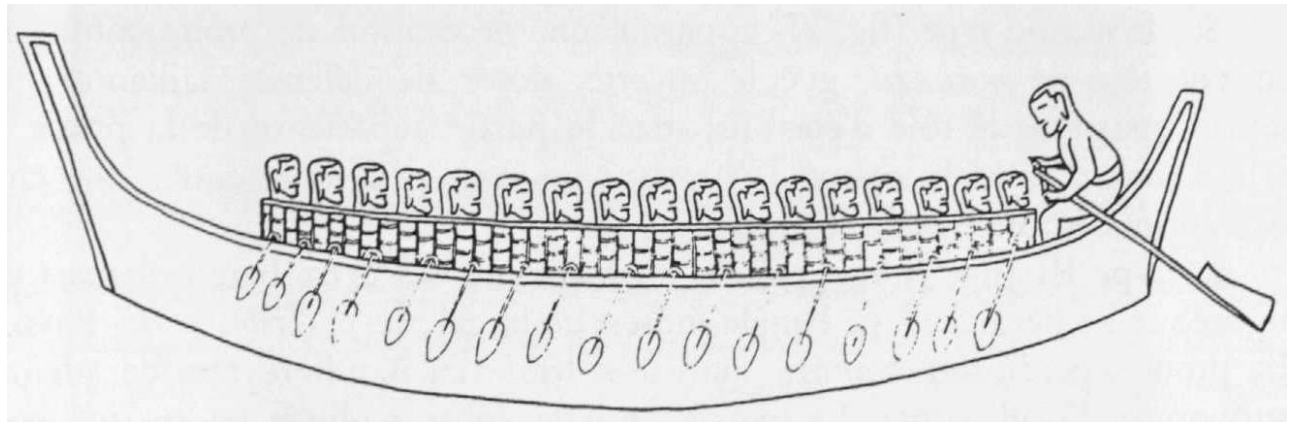

Figure 1. Barque de guerre khmère. Type I. Bàyon : Gal. Ext., Face S., Aile E, Partie Inf. (Dessin M. J.-H.)

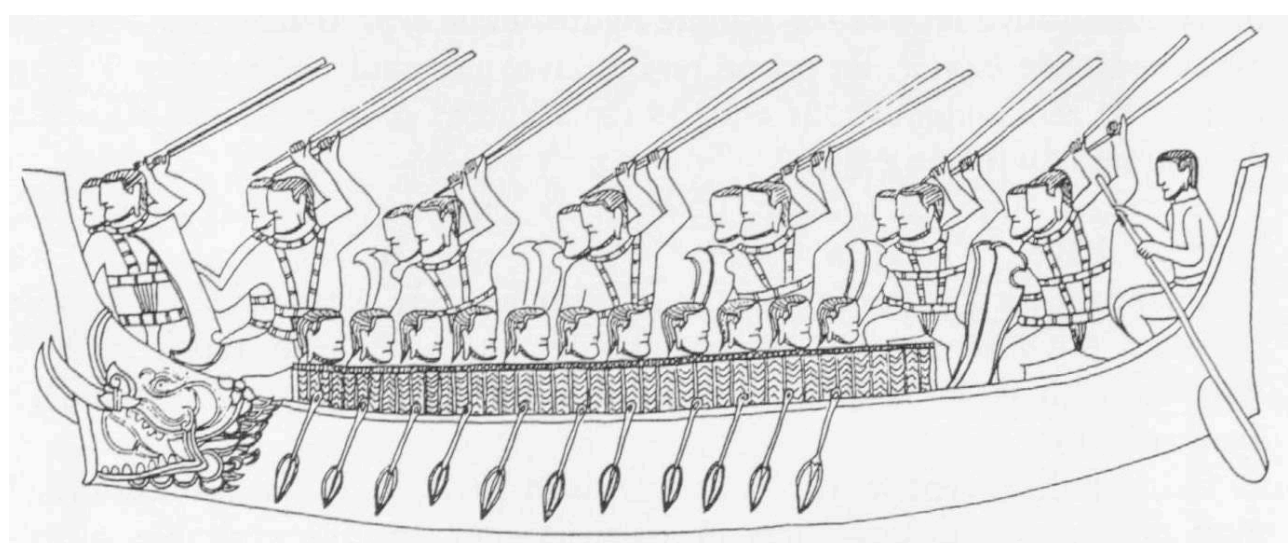

Figure 2. Barque de guerre khmère. Type II. Bàyon : Gal. Ext., Face S., Aile E., Partie Inf. (Dessin M. J.H.) 


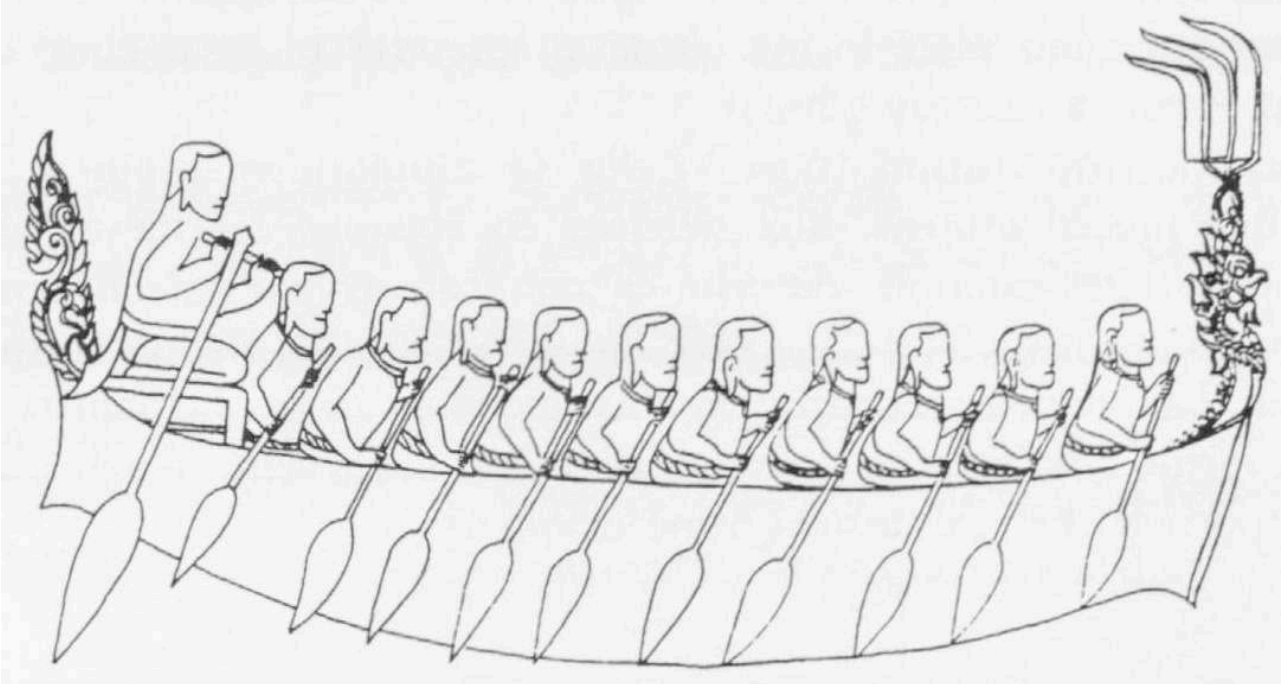

Figure 3. Barque de guerre khmère. Type III. Bàyon : Gal. Ext., Face S., Aile E. (Dessin M. J.-H.)

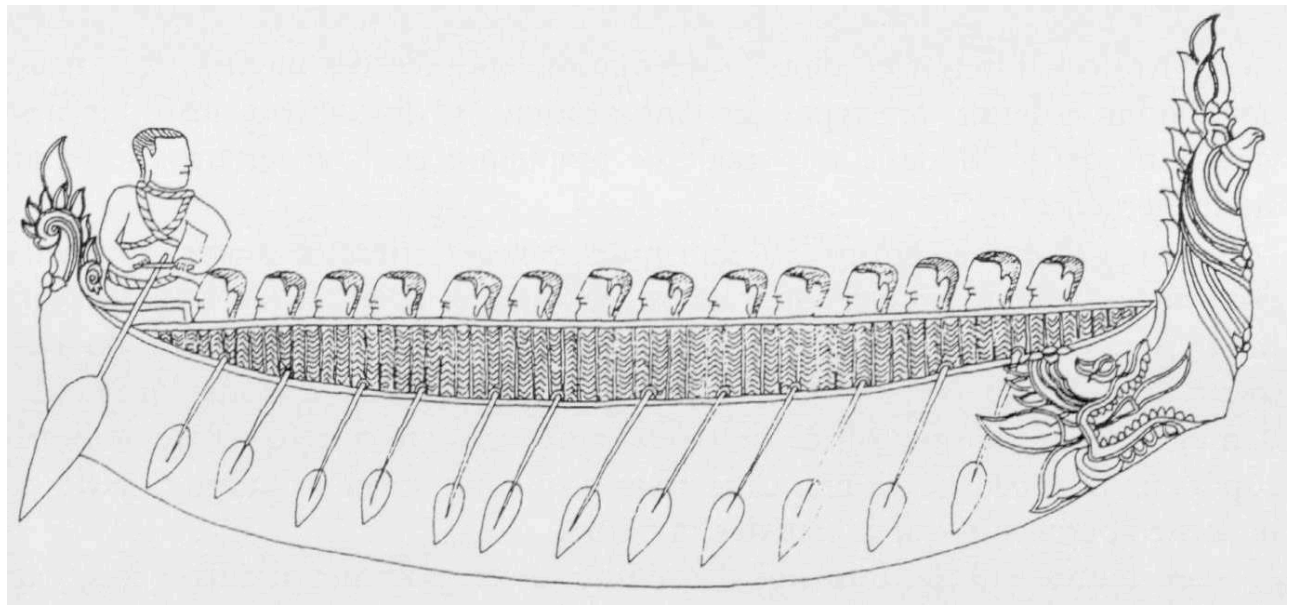

Figure 4. Barque de guerre khmère. Type IV. Bàyon : Gal. Ext., Face S., Aile E., Partie Inf. (Dessin M. J.H.)

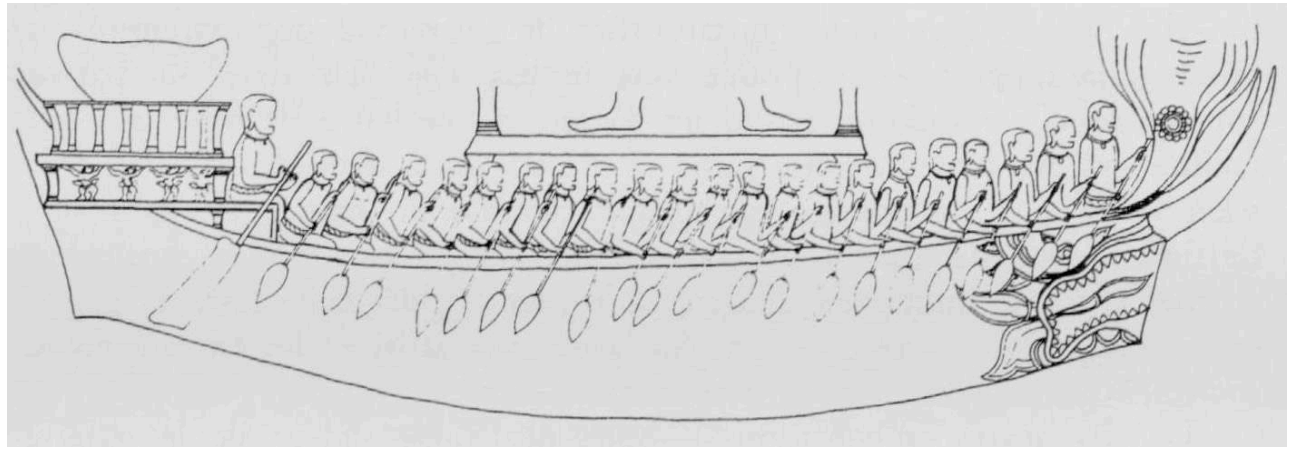

Figure 5. Barque amirale khmère. Type V. Bàyon : Gal. Ext., Face S., Aile E., Partie Sup. (Dessin M. J.-H.) 


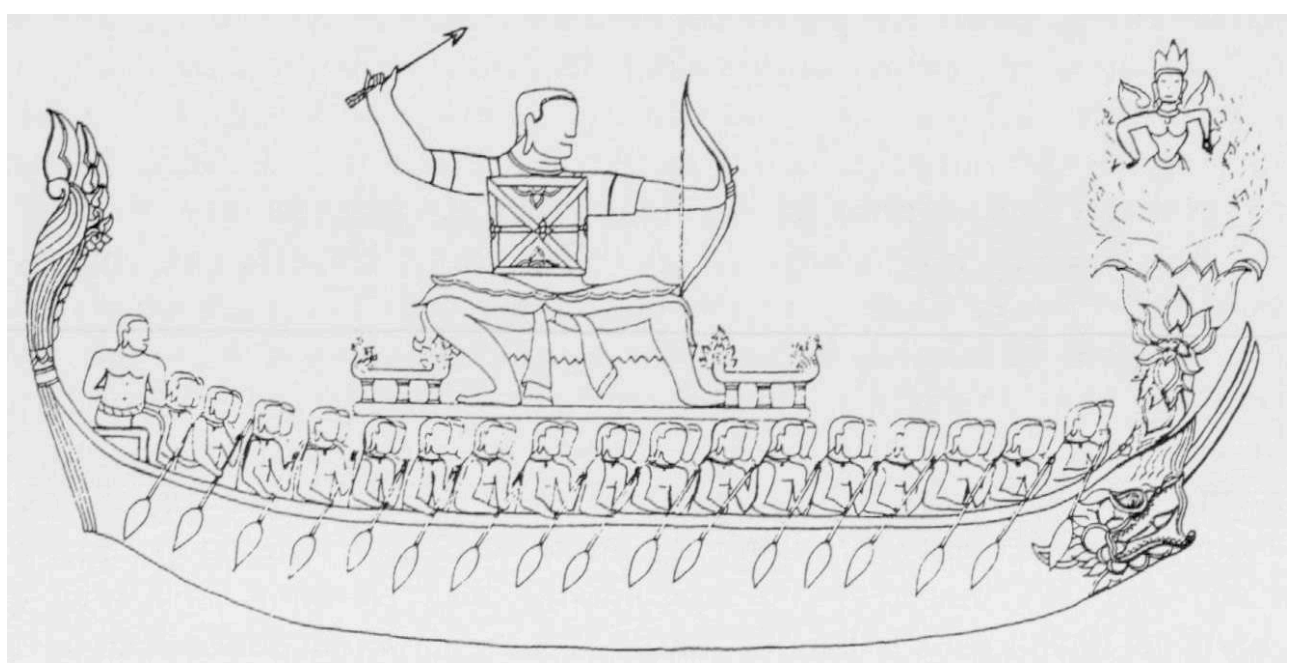

Figure 6. Barque amirale khmère. Type VI. Banteay Chmar : Gal. E., Partie Sup. (Dessin M. J.-H.) barques khmères, les représentations en sont plus ou moins complètes ; une dizaine 
d'entre elles peut être vraiment étudiée : leur forme ne varie pas de celle des bateaux khmers et leur décoration reprend les mêmes thèmes. Le type le plus représenté est le type II khmer (fig. 2), c'est-à-dire la tête de makara simple en proue, associée à une poupe lisse. Six embarcations comportent cette décoration. Nous en avons isolé une, qui figure au Bàyon dans la bataille navale, à cause de son étambot incliné vers l'avant (fig. 7).

Trois embarcations cames sont proches du type IV khmer. Elles sont au Bàyon, mais à part de la bataille navale, plus à l'ouest, et participent à une sorte d'attaque isolée des positions khmères (fig. 8) ; leurs occupants s'apprêtent à débarquer et, plus loin, rencontrent les guerriers khmers. Venant après la grande bataille navale, cette scène évoque peut-être une dernière tentative des Cams pour reprendre l'avantage après leur défaite. À la différence du type IV khmer, leurs décorations sont infiniment plus soignées, et leurs sculptures très riches.

21 Enfin, existe le type unique d'un navire cam de la bataille du Bàyon (fig. 9) parce qu'à sa décoration de type II s'ajoute, coiffant la proue, une petite cabine, peu différente de celle qui figurait en poupe sur l'embarcation khmère de type $V$ (fig. 5).

Toutes ces embarcations cames sont déplacées à la rame ; les rameurs, protégés par des claies de rotin, font face à l'arrière, comme les Khmers, sauf pour trois embarcations de Banteay Chmar dont nous avons déjà fait état. La direction des navires est aussi assurée par des nautoniers placés à l'arrière et munis de la même grande rame déjà observée sur les bateaux khmers. Ces similitudes entre les embarcations cames et leurs rivales khmères tendraient à prouver qu'il ne s'agit pas de véritables copies de modèles précis.

C'est là tout ce que l'on peut objectivement dire des barques qui figurent sur les basreliefs de ces deux temples khmers. Il faut, à partir de là, extrapoler sur ce qu'étaient leurs dimensions réelles, les matériaux qui entraient dans leur fabrication et la manière dont ils étaient construits.

À propos de leurs dimensions, un texte datant des Qi méridionaux (479-502 AD) que cite P. Pelliot à propos du Funan (1903: 261) fournit les détails suivants : «On fait des bateaux de 8 à 9 tchang. On les taille en largeur à 6 ou 5 pieds. L'avant et l'arrière sont comme la tête et la queue d'un poisson ». G. Groslier (1921:111) donne, comme correspondance à ces mesures chinoises, 24 à $25 \mathrm{~m}$ de longueur, 1,5 à 1,8 $\mathrm{m}$ de largeur.

Si l'on essaie de se faire une idée de leur taille en comptant les rameurs ou les pagayeurs, ou encore le nombre de rames ou de pagaies, on se heurte à une difficulté sérieuse : il est bien rare, en effet, que les deux effectifs correspondent pour une même embarcation. Très souvent, le nombre des rameurs est supérieur à celui des rames visibles. P. Paris (1941) arrive à des moyennes de 13 avirons de chaque bord. Si l'on admet qu'un mètre au moins sépare chaque rameur, cela fait 12 intervalles d'un mètre, auxquels il faut ajouter les extrémités avant et arrière. P. Pâris estime que l'on peut alors évaluer la longueur du bateau à 17 mètres au minimum. Mais on s'aperçoit, sur certains exemples, que les sculpteurs, pris peut-être d'un souci de vérité, ont tassé jusqu'à 23 avirons dans un espace primitivement conçu pour 15. 


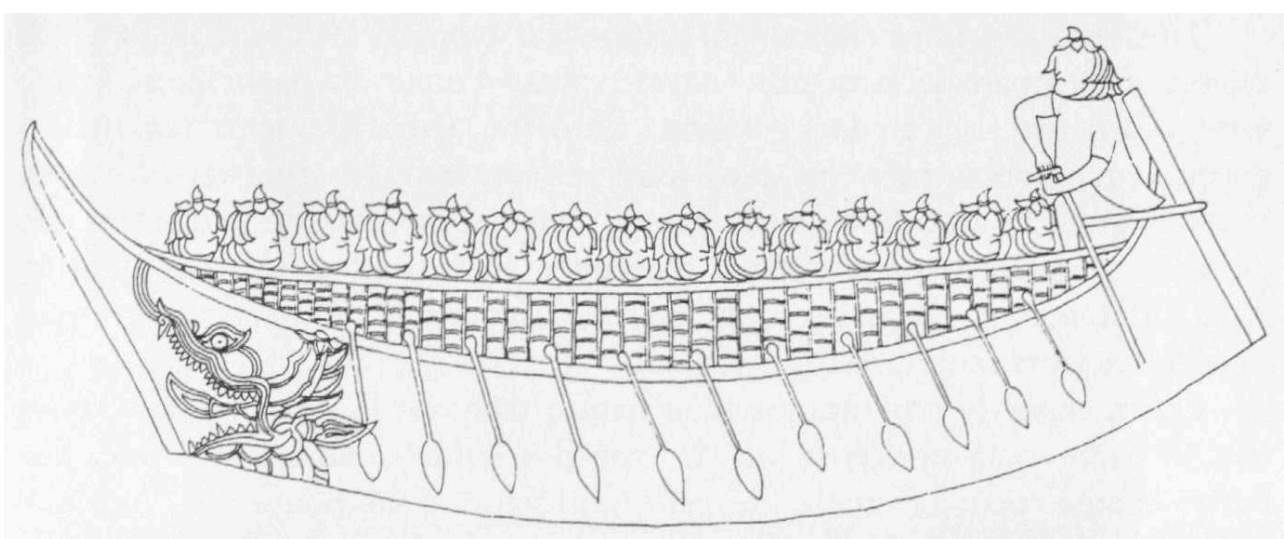

Figure 7. Barque de guerre came Bàyon : Gal. Ext., Face S., Aile E., Partie Inf. (Dessin M. J.-H.)

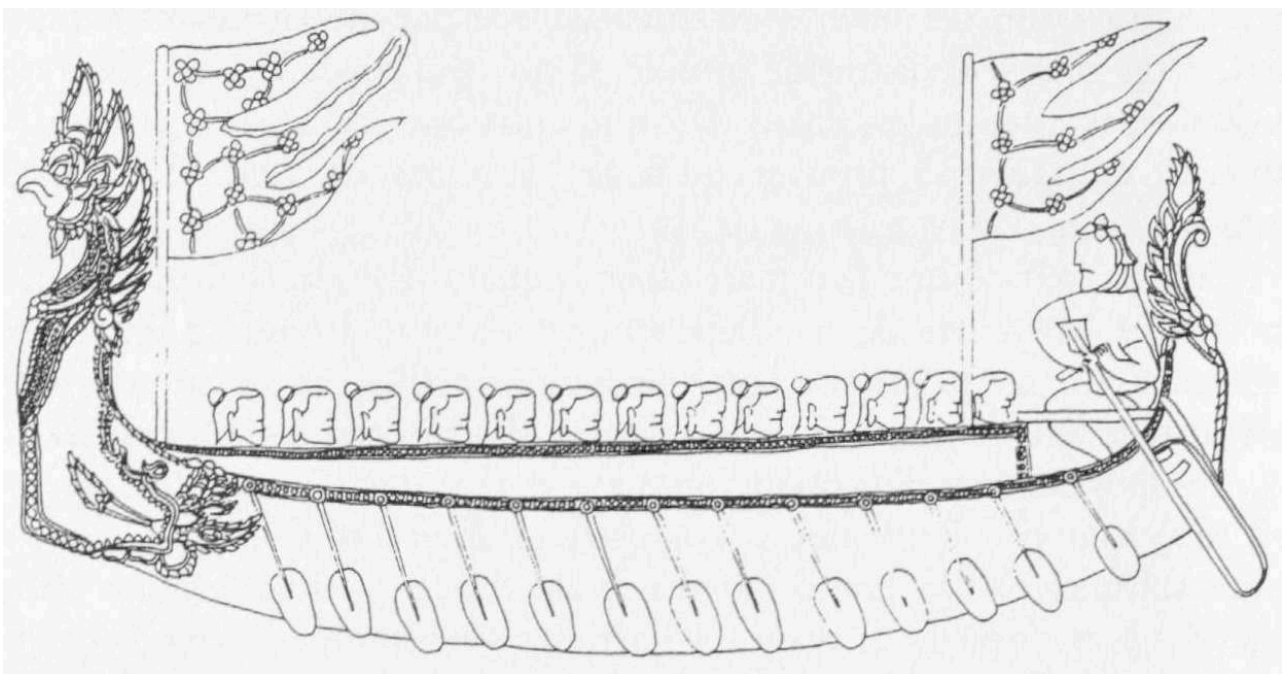

Figure 8. Barque de guerre came. Bàyon : Gal. Ext., Face S., Aile E, Reg. Inf. (Dessin M. J.-H.)

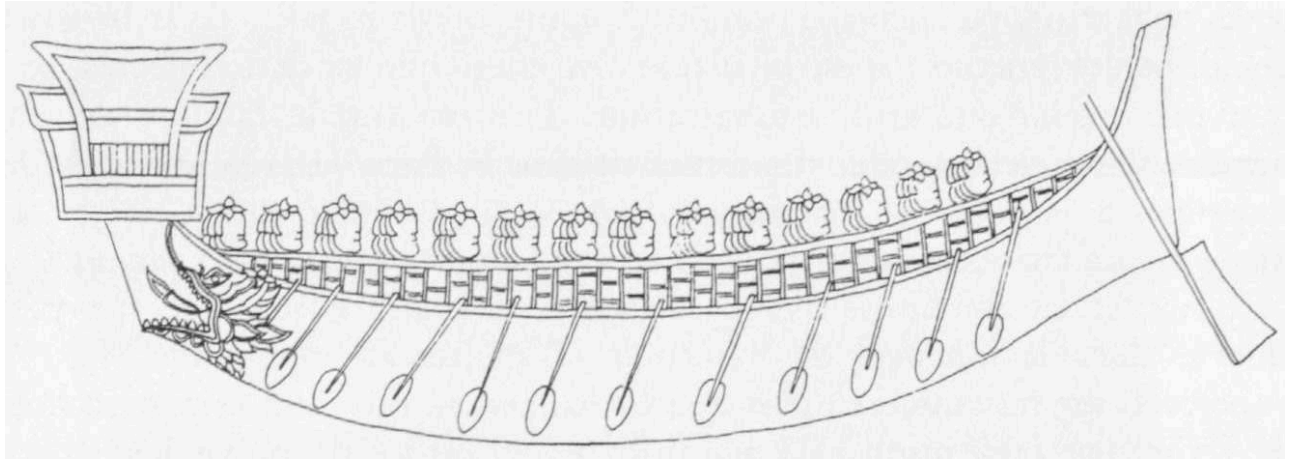

Figure 9. Barque de guerre came. Bàyon : Gal. Ext., Face S., Aile E., Partie Sup. (Dessin M. J.-H.)

Si l'on retient ce chiffre de 23 avirons, les $27 \mathrm{~m}$ de longueur proposés par G. Groslier (1921) peuvent être atteints. Pour les barques conduites à la pagaie, Paris précise que la même incertitude persiste. L'intervalle entre les pagayeurs peut être ramené à $0,80 \mathrm{~m}$. La barque amirale du Bàyon comportant 21 pagayeurs (c'est le maximum qui nous est proposé) : cela donne une longueur de $21 \mathrm{~m}$ au moins. Paris ajoute que les pirogues du Cambodge moderne comportent 15 pagayeurs de chaque bord et que leur longueur, est néanmoins de 20 à $21 \mathrm{~m}$, d'où le fait qu'il serait peut-être nécessaire d'augmenter les dimensions proposées, notamment à l'avant et à l'arrière. Quoi qu'il en soit, les 
embarcations mues à la pagaie de l'angle sud-est mises à part, les deux barques amirales déplacées de cette manière, au Bàyon d'une part, à Banteay Chmar d'autre part, paraissent au moins une fois et demie plus grandes que leurs voisines pourvues d'avirons.

Ces barques étaient toutes monoxyles; et il est fort peu probable qu'elles furent faites de planches assemblées et calfatées, car le détail des joints aurait alors été figuré par les sculpteurs, au moins sur quelques exemplaires. Or, la seule embarcation qui, dans l'ensemble des bas-reliefs des deux temples montre ce détail de construction (galerie sud, aile est du Bàyon), est totalement différente. C'est une sorte de jonque de mer avec de hauts bordages. Si l'on rappelle à ce propos le témoignage de Zhou Daguan (Tcheou Ta-kouan) (Pelliot: 1902: 172), nous apprenons que « les petites barques sont faites d'un grand arbre qu'on creuse en forme d'auge : on l'amollit au feu et on l'élargit par effort de pièces de bois; aussi ces barques sont-elles larges au centre et effilées aux deux bouts ». Groslier (1921: 111), constatant qu'il y a similitude entre les embarcations évoquées dans les textes ou figurées sur les bas-reliefs et les embarcations de ce type au Cambodge moderne, avance que les procédés de fabrication ont dû aussi rester les mêmes. Il est ainsi conduit à énumérer un certain nombre d'espèces d'arbres qui servaient en son temps à cette fabrication: le koki (bois dur inattaquable à l'eau et aux vers), le khlong, le pôpél, le khnong, le maysac. Il ajoute que tous ces arbres atteignent de grandes tailles et que des pirogues presque sans défaut peuvent sortir d'un fût de $35 \mathrm{~m}$ de long. De plus, écrit-il, on ne peut guère parler de matériau de calfatage à propos de ces embar-cations, mais peut-être étaient-elles enduites du vernis noir brillant alors employé au vernissage des coques. Cet enduit était fabriqué à partir de la résine d'un arbre appelé krul, mélangée à des oléo-résines désignées par le nom générique de chêar-toek. Il est par ailleurs possible -et même probable- que les figures sculptées des proues et des poupes étaient peintes de couleurs vives ou dorées.

Toutes ces barques sont surchargées de guerriers armés jusqu'aux dents: de lances pour la plupart, plus rarement d'arcs et de flèches (fig. 2). Ils sont disposés sur un ou deux rangs, parfois sur trois, et se tiennent debout entre les deux rangées de rameurs sur un espace que l'on peut appeler la coursive. Leur nombre varie de 15 à 20 . Nous avons déjà remarqué, sur les deux navires amiraux, que le commandant se tenait sur une estrade surélevée qu'on a pu rapprocher (Paris 1941:339) de celle où se tient l'archer des pirogues gravées sur des tambours de bronze étudiés pour la première fois par V. Goloubew (1929: 34, Pl. XXVII) (fig. 10 A, B). Le rapprochement est en effet tentant et Paris (1941: 357) écrit: " [1']objection que la superstructure où se tient l'archer rendrait instable toute embarcation monoxyle ou ayant les proportions d'une pirogue n'est pas décisive ", car, dit-il, « une pirogue monoxyle déployée au feu et à l'eau peut atteindre $1,8 \mathrm{~m}$ de large, $1,4 \mathrm{~m}$ à la flottaison. Sa section est sensiblement un arc de cercle très évasé prolongé au-dessus de la flottaison par deux bouts de tangentes. 


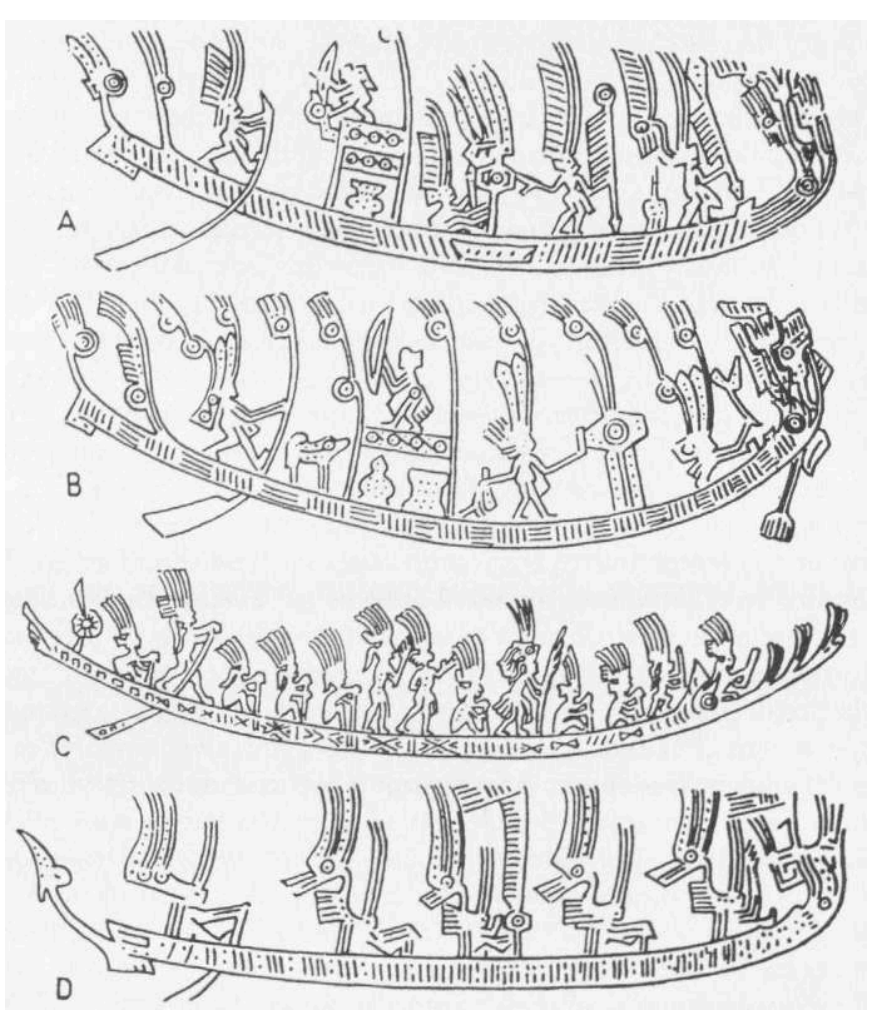

Figure 10. Barques de tambours de bronze dongsonniens (Goloubew 1940 : fig 15) tambours de bronze dits de Dông-so'n. Ce toponyme renvoie à un site proche du village du même nom, situé sur la rive droite de la Sông-mâ, province de Tranh-hoa, dans la région sud du delta de la Rivière Rouge au Nord Viêt Nam qui, à la suite de nombreuses fouilles durant l'entre-deux-guerres, révéla de nombreux objets en bronze, parmi lesquels des tambours. On parla alors de civilisation de Dông-so'n, mais depuis longtemps on connaissait des tambours identiques ou similaires à ceux de ce site dans toute l'Asie du Sud-Est, y compris en Indonésie et en Chine méridionale. La première étude fondamentale qui leur fut consacrée fut celle de F. Heger (1902), qui établit une classification des 165 tambours alors connus et les répartit en 4 catégories, à savoir Heger I, II, III, IV, en tenant compte de leur allure générale, des accessoires dont ils pouvaient ou non être pourvus (anses, grenouilles en ronde-bosse), et de leur décor gravé, naturaliste ou abstrait. Cette classification a été conservée et «il est généralement admis que ces types se suivent non seulement d'une façon chronologique mais aussi génétique ». (Loofs-Wissowa 1992 : 194). Depuis lors, de nombreuses autres pièces ont été découvertes: plus de 400 sont bien connues et l'on sait que la Chine méridionale en a révélé encore bien plus. appartiennent à la première catégorie, Heger I. En effet, c'est uniquement sur quelque $20 \%$ au plus des pièces de celle-ci qu'apparaissent des figurations de barques, uniquement sur la surface bombée du tambour qui unit le disque à la caisse. Elles constituent un des éléments du décor gravé qui a donné lieu au plus grand nombre 
d'interprétations, avec les figurations d'oiseaux stylisés en plein vol, les silhouettes de guerriers emplumés et la supposée "étoile " centrale du disque, ces interprétations allant évidemment de pair avec le rôle qu'on voulait assigner à ces mystérieux tambours. Comme le fait remarquer malicieusement H. Loofs-Wissowa (1992: 193), ce «sont, en effet, des objets rituels par excellence puisque personne ne sait exactement comment ils ont été utilisés aux temps dongsonniens, et il est bien connu que si un archéologue ne sait pas comment interpréter un objet il l'appelle "rituel" ».

Ce n'est pas le lieu ici d'entrer dans le débat sur leur signification véritable. Faisons simplement état des derniers travaux publiés sur cette question par H. Loofs-Wissowa lui-même $(1983,1991,1992)$, dont le bon sens et le réalisme de l'analyse nous ont beaucoup séduit. Il en ressort que ces tambours ne peuvent plus être considérés comme les instruments privilégiés de quelque chamanisme, comme cela a pu être avancé à partir d'hypothèses biscornues sur d'éventuelles influences religieuses, réciproques en ce domaine, entre l'Asie du Sud-Est septentrionale et la Sibérie ou même l'Asie Centrale septentrionale. Cet auteur montre de manière convaincante que la figuration de l'« étoile» centrale commune à tous les tambours -sur laquelle s'appuie largement l'hypothèse du chamanisme- n'en était pas une, pas plus qu'un quelconque astre, mais le résultat du vide obligé qu'implique la disposition concentrique du motif en triangles en plus ou moins grand nombre au centre du tympan, motif omniprésent dans le décor de ces objets. Mais, bien que non liés à une religion particulière, ces tambours eurent une signification pour les populations du Sud-Est asiatique qui s'en pourvurent, car les pièces de type I, au moins, ont toutes la même provenance. Il est en effet désormais admis qu'ils ne peuvent avoir été fabriqués localement sur les lieux approximatifs de leur découverte ; l'évolution de la métallurgie du bronze ne le permettait pas. En outre, l'uniformité de leur décoration prêche fortement pour cette origine unique. Celle-ci ne serait pas aussi frappante si des "imitations " avaient été faites ici et là à partir de lointains modèles. H. Loofs-Wissowa montre que les deux motifs principaux de ce décor, l'oiseau surtout, et la barque, renvoient en fait à un environnement qui correspond à celui de la péninsule indochinoise septentrionale, autrement dit au Nord Viêt Nam, avec Dông-so'n. L'oiseau, un probable héron, serait une sorte d'animal héraldique des populations de cette région, créature aquatique associée au concept de fertilité qui, en Asie du Sud-Est, est indissociable de l'eau. Quant aux barques, ce sont à l'évidence des embarcations fluviales et les personnages emplumés qu'elles transportent sont probablement les participants de quelque rituel de fertilité présidé par une autorité politico-religieuse, tradition qui s'est perpétuée jusqu'à notre époque dans quelques royautés du Sud-Est asiatique (Laos, Thaïlande).

Ces tambours furent donc fabriqués dans une même région et diffusés dans toute l'Asie du Sud-Est par le biais des réseaux de commerce qui liaient les différentes contrées. Est-ce à dire qu'ils ne constituèrent qu'un objet de ce commerce parmi d'autres (produits alimentaires, poteries, outils divers). H. Loofs-Wissowa en doute et propose la "Regalia Theory " pour expliquer leur diffusion. Ces objets auraient été par essence trop sacrés, en tant qu'instruments d'autorité, pour constituer un banal produit d'échange. Ils auraient été accordés par une autorité spirituelle résidant en quelque lieu de l'Indochine septentrionale (pas nécessairement Dông-so'n) à des chefs sud-est asiatiques.

«We might thus imagine the coming and going of embassies or missions from tribal chiefs in various parts of Southeast Asia who through the obtaining of a drum would seek to become kings in the then accepted sense of the term and thus be 
integrated into a wider politico-religious system, transcending their own relatively limited one. Or, alternatively, we may imagine the sending of emissaries from the "Holy See" to take these drums to far-away places for the same purpose. » (1991 : 47).

$34 \mathrm{Au}$ vu de cette nouvelle interprétation, les "barques magiques", les «barques des morts» dont ont complaisamment parlé certains auteurs en les rapprochant de pratiques étrangères à leur milieu d'origine -telles celles des Dayaks de Bornéo-, sont ramenées au rôle de barques fluviales ordinaires employées, à l'occasion, lors de cérémonials de fertilité.

Elles sont donc représentées avec beaucoup de schématisme mais toutes sur le même modèle (fig. 10). La coque, dont il n'y a pas lieu de douter qu'elle était monoxyle, s'incurve en forme de croissant, la proue s'ornant de figures animales dressées (fig. 11), tandis que la poupe se relève aussi sous l'apparence fréquente d'une queue d'animal fantastique (fig. 10); un nautonier pourvu d'une longue rame faisant office de gouvernail se tient aussi à l'arrière, tandis que, beaucoup plus rarement, des pagayeurs sont répartis au long des flancs (fig. $10 \mathrm{C}$ ).

Rien, comme on le voit, qui diffère fondamentalement de ce que nous avons décrit avec des détails plus précis à propos des barques khmères. La présence de personnages aux coiffures emplumées debout dans la barque ou juchés sur une estrade au-dessous de laquelle on découvre parfois la silhouette d'un vase cérémoniel ou d'un tambour (fig. 10 A, B), cela en l'absence de tout pagayeur, a largement contribué à alimenter les théories les plus fumeuses sur les tambours de bronze et leur usage. Il ne s'agit là, bien sûr, que de simplifications et de schématisations.

Les tambours de bronze de type Heger I, porteurs, éventuellement, de ces figurations de barques monoxyles aux proues et aux poupes ornées, mues à la pagaie et dirigées au gouvernail, sont les plus anciens. Leur datation, toutefois, n'a jamais cessé d'être l'objet de controverses, les appréciations sur celle-ci allant du IX ${ }^{\mathrm{e}}$ au $\mathrm{I}^{\mathrm{er}}$ siècle avant J.-C. Elles ne sont donc que les très lointains ancêtres des barques de guerres khmères ou cames des bas-reliefs du Bàyon et de Banteay Chmar, mais la filiation est indéniable. 


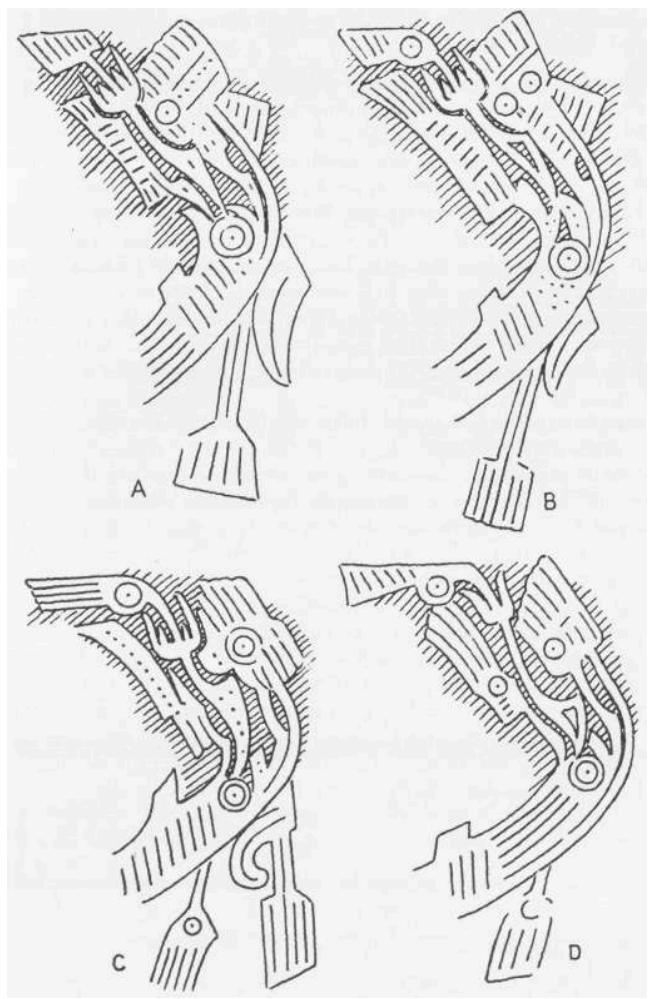

Figure 11. Figures de proue de barques de tambours de bronze dongsonniens (Goloubew 1940 : fig. 17)

\section{BIBLIOGRAPHIE}

Cœdes, Georges, 1929, « Études cambodgiennes. XXIV. Nouvelles données chronologiques et généalogiques sur la dynastie de Mahîdharapura », Bulletin de l'École Française d'Extrême-Orient (BEFEO) XXIX : 297-330.

- 1932, «Études cambodgiennes. XXVIII. Quelques suggestions sur la méthode à suivre pour interpréter les bas-reliefs de Banteay Chmar et de la galerie extérieure du Bàyon ", Bulletin de l'École Française d'Extrême-Orient (BEFEO) XXXII : 71-81.

Goloubew, Victor, 1929, « L'âge du bronze au Tonkin et dans le Nord-Annam », Bulletin de l'École Française d'Extrême-Orient (BEFEO) XXIX : 1-46.

1940, « Le tambour métallique de Hoàng-ha », Bulletin de l'École Française d'Extrême-Orient (BEFEO) XL : 383-409.

Groslier, Georges, 1921, Recherches sur les Cambodgiens d'après les textes et les monuments depuis les premiers siècles de notre ère. Paris.

Heger, F., 1902, Alte Metalltrommeln aus Südost-Asien. Texband, Tafelband. Leipzig : H. Hiersemann. Jacq-Hergoualc'h, Michel, 1979, L'armement et l'organisation de l'armée khmère d'après les bas-reliefs d'Angkor Vat, du Bàyon et de Banteay Chmar. Paris : Presses Universitaires de France. 
Loofs - Wissowa, Helmut, 1983, The Distribution of Dongson Drums : some Thoughts », pp. 410-417, in P. Snoy (ed.), Ethnology und Geschichte : Festschrift für Kart Jettmar. Wiesbaden. - 1991, « Dongson Drums : Instruments of Shamanism or Regalia?», Arts Asiatiques XLVI : 39-49. - 1992, « Les tambours de bronze dits de Dongson et les astres », pp. 192-218, in Disciplines croisées. Hommage à B.-Ph. Groslier 1. Paris : EHESS

Paris, Pierre, 1941, « Les bateaux des bas-reliefs khmers », Bulletin de l'École Française d'ExtrêmeOrient (BEFEO) XLI : 335-361.

Pelliot, Paul, 1902, « Mémoires sur les coutumes du Cambodge de Tcheou Ta-kuan (XIII ${ }^{\mathrm{e}}$ siècle) », Bulletin de l'École Française d'Extrême-Orient II : 123-177.

- 1903, « Textes des Ts'i Méridionaux dans l'article "Le Founan" 2 », Bulletin de l'École Française d'Extrême-Orient III : 248-303.

\section{RÉSUMÉS}

Seront évoquées ici les modalités de deux occurrences des représentations relatives aux embarcations monoxyles, faites à des époques fort différentes et sur des subjectiles très dissemblables. Celles, d'une part, qui apparaissent sur les tambours de bronze de la civilisation de Dông-so'n (Viêt Nam), d'autre part, certaines de celles que l'on découvre dans les bas-reliefs de deux grands temples khmers datés de la fin du XII ${ }^{\mathrm{e}}$ ou du début du XIII ${ }^{\mathrm{e}}$ siècle.

We will evoke the modalities of two occurrences that were determining factors of representations, relative to monoxyla boats built during different epochs and from different supports. On the one hand, those found on the bronze drums of the Dong So'n (Viet Nam) civilization and those discovered on the bas-reliefs of two important khmer temples dating from the end of the XII ${ }^{\text {nd }}$ century or the beginning of the XIII ${ }^{\text {rd }}$ century.

Serán evocadas aquí las modalidades de dos ocurrencias de las representaciones relativas a las embarcaciones monoxilas hechas en épocas completamente diferentes y sobre soportes muy desiguales. Por una parte, las que aparecen sobre los tambores de bronce de la civilización Dông So'n (Viet Nam); por otra parte, algunas de las que se descubren en los bajorrelieves de dos grandes templos kmer datando de fines del siglo XII o principios del XIII.

\section{INDEX}

Mots-clés : barque monoxyle, tambour de bronze, bas-reliefs khmers, Dông-so'n, Bàyon, Banteay Chmar, Viêt Nam

\section{AUTEUR}

\section{MICHEL JACQ-HERGOUALC'H}

Directeur de recherche, CNRS - UPRES-A 7019, Institut d'Extrême-Orient du Collège de France, 52 rue du Cardinal Lemoine, 75005 Paris 